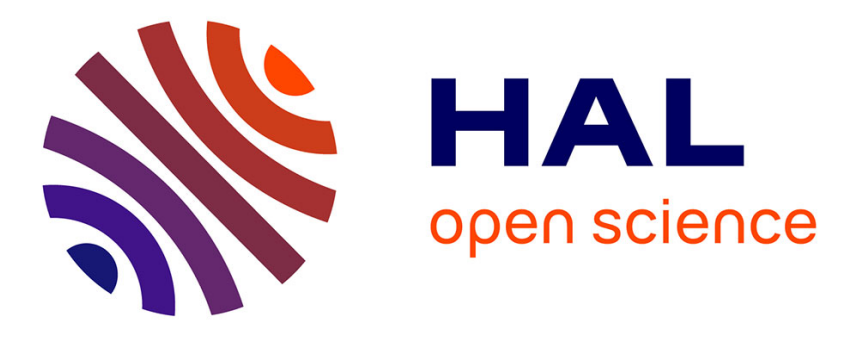

\title{
Structural study of amorphous polyaniline
}

\author{
M. Laridjani, Jean Pouget, A. Macdiarmid, A. Epstein
}

\section{To cite this version:}

M. Laridjani, Jean Pouget, A. Macdiarmid, A. Epstein. Structural study of amorphous polyaniline. Journal de Physique I, 1992, 2 (6), pp.1003-1010. 10.1051/jp1:1992193 . jpa-00246581

\section{HAL Id: jpa-00246581 https://hal.science/jpa-00246581}

Submitted on 1 Jan 1992

HAL is a multi-disciplinary open access archive for the deposit and dissemination of scientific research documents, whether they are published or not. The documents may come from teaching and research institutions in France or abroad, or from public or private research centers.
L'archive ouverte pluridisciplinaire HAL, est destinée au dépôt et à la diffusion de documents scientifiques de niveau recherche, publiés ou non, émanant des établissements d'enseignement et de recherche français ou étrangers, des laboratoires publics ou privés. 
Classification

Physics Abstracts

$61.40 \mathrm{~K}$

\title{
Structural study of amorphous polyaniline
}

\author{
M. Laridjani ( ${ }^{1}$ ), J. P. Pouget ( $\left.{ }^{1}\right)$, A. G. MacDiarmid ( ${ }^{2}$ ) and A. J. Epstein ( ${ }^{3}$ ) \\ (1) Laboratoire de Physique des Solides, Université de Paris-Sud, 91405 Orsay, France \\ (2) Department of Chemistry, University of Pennsylvania, Philadelphia, PA 19104-6323, U.S.A. \\ (3) Department of Physics and Department of Physics, The Ohio State University, Columbus, \\ Ohio 43210-1106, U.S.A.
}

(Received 31 January 1992, accepted 17 March 1992)

\begin{abstract}
Résumé. - Beaucoup de matériaux, spécialement les polymères, ont une importante fraction de leur volume sans ordre cristallin à longue portée. Bien que ces régions soient souvent appelées amorphes, elles présentent fréquemment un ordre local caractéristique. Nous décrivons et utilisons dans ce papier une méthode, basée sur une technique de diffraction de rayons $\mathrm{X}$ non dispersive en énergie, pour obtenir des fonctions d'interférence de bonne qualité et, par transformée de Fourier, la fonction de distribution radiale des polymères amorphes. Nous appliquons cette technique à plusieurs éléments d'une même famille de polymères électroniques d'intérêt actuel : les polyanilines éméraldine bases. Nous montrons que l'ordre local présente d'appréciables différences dans les matériaux de type I et II, préparés respectivement sous forme de sel et de base. Cette étude démontre l'importance des conditions de préparation sur les propriétés physiques du polyaniline et donne une base structurale aux effets observés dans les processus de dopage-dédopage de ces matériaux.
\end{abstract}

\begin{abstract}
Many materials, especially polymers, have a substantial volume fraction with no long range crystalline order. Though these regions are often termed amorphous, they frequently have a specific local order. We describe and use here a method, based on a non-energy dispersive X-ray diffraction technique, to obtain good quality interference functions and, by Fourier transform, radial distribution functions of the amorphous structure of polymers. We apply this approach to members of a family of electronic polymers of current interest : polyaniline emeraldine bases. We show that the local order exhibits significant differences in type I and type II materials, precipitated as salt and base respectively. These studies demonstrate the importance of sample preparation in evaluating the physical properties of polyaniline, and provide a structural origin for memory effects observed in the doping-dedoping processes.
\end{abstract}

\section{Introduction.}

The X-ray diffraction method has been extensively applied to determine the structure of amorphous solids. Structural information can « only » be obtained by the Fourier transform of the coherent part of the diffracted intensity [1]. To obtain this component the subtraction

(*) URA 02 - CNRS. 
of an incoherent scattering background, mainly due to Compton scattering. from the total scattered intensity is required. It is a major problem to assess the intensity of the coherent component at sufficiently large $K$ wave vectors $(K=4 \pi \sin \theta / \lambda)$, because for such $K$ values the coherent intensity is generally weaker than the Compton intensity. This is especially the case for low atomic number substances such as organic polymers. Thus the difficulty in obtaining the coherent diffracted intensity explains why there are relatively few $X$-ray determinations of local order in amorphous polymers available in the literature [2]. It is therefore desirable to remove the incoherent scattering during the experimental measurement itself as effectively as possible. Such an elimination is facilitated by the use of a non-energy dispersive $X$-ray diffraction method [3]. We have recently applied [4] with success such a method to determine the local order in several amorphous forms of a family of electronic polymers of current interest : polyaniline [5]. Here we extend this work to another (type I) class of polyanilines which differs from the previously studied [4] type II class of materials.

Depending upon the method of preparation, two classes of the emeraldine form of polyaniline can be distinguished [6,7]. Class I consists of materials prepared in the conducting doped emeraldine salt, ES (Fig. 1b), form, while Class II consists of materials prepared in the insulating emeraldine base, EB (Fig. 1a), form. As prepared hydrochloride ES-I films and powders are substantially « crystalline ». Dedoping of ES-I leads to essentially amorphous emeraldine base EB-I. Redoped EB-I yields back the substantially «crystalline » EB-I. Depending on preparation conditions EB-II can be either substantially « crystalline » (case of powders) or completely amorphous (case of most of the unstretched films and fibers). Upon doping with $\mathrm{HCl}, \mathrm{EB}-\mathrm{II}$ is converted into ES-II which can be, dependent upon the original base structure, either partially « crystalline » or completely amorphous, but dedoped ES-II is always amorphous.

(a)<smiles>[X]C(C)c1ccc(N=C2C=CC(=Nc3ccc(Nc4ccc(N(C)C(C)C)cc4)cc3)C=C2)cc1</smiles><smiles>[X]C(C)c1ccc(N=[NH+]c2ccc(N=[NH+]c3ccc(N([TlH])CC)cc3)cc2)cc1</smiles>

Fig. 1. - Schematic illustration of the polymer repeat unit for : (a) polyaniline emeraldine base (EB); (b) polyaniline emeraldine $\mathrm{HCl}$ salt (ES).

The structure of the «crystalline " part of EB-II, ES-I and ES-II has been derived from comparison between the diffraction pattern and intensity calculations [7]. The average EB-II structure, figure $2 \mathrm{a}$, is similar to that of chemically analoguous polymers such as poly( $\mathrm{p}$ phenylene sulfide) and poly(p-phenylene oxide). The average phenyl ring tilt angle, with respect to the plane of nitrogens, is $\Phi \sim 30^{\circ}$ and the average ring- $\mathrm{N}$-ring angle is $\delta \sim 130^{\circ}$. An approximate representation of the hydrochloride ES-I structure is shown figure $2 \mathrm{~b}$. It is found that the rings are nearly in the plane of the nitrogens $\left(\Phi \sim 0-15^{\circ}\right)$. The 


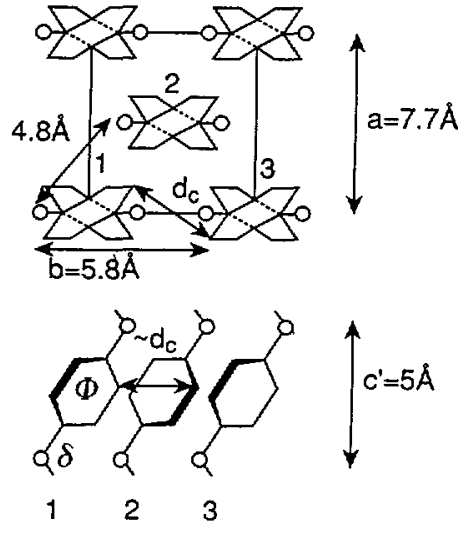

a)

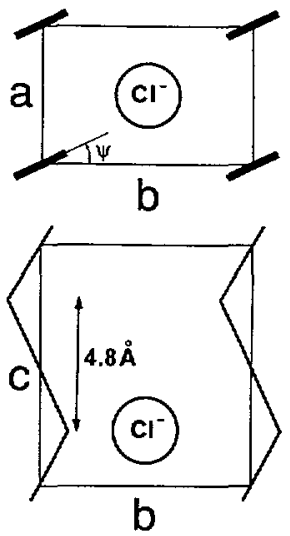

b)

Fig. 2. - Projection along the chain axis and side view of the EB-II (a) and ES-I (b) structures.

polymer chain setting angle is estimated at $\psi \sim 20-30^{\circ}$, and the chains are probably tilted with respect to the $(a, b)$ basal plane. Hydrochloride ES-II and ES-I differ from one another in structure [6,7]. The hydrochloride ES-II structure is described in reference [7].

A recent non-energy dispersive X-ray diffraction study [4] has shown that amorphous EB-II and ES-II unstretched films exhibit local chain array resembling those shown by the EB-II and ES-II « crystalline » structures respectively. This conclusion has been obtained from the close correspondence between the average interatomic distances deduced from the radial distribution functions and distances calculated from the EB-II and ES-II « crystalline » structures. It was also found that dedoped unstretched ES-II film has an increased interchain disorder though a local structural order intermediate between those of amorphous EB-II and ES-II. Here we report a similar investigation of amorphous EB-I powder, obtained by dedoping of partially "crystalline » ES-I powder, in order to compare the EB-I local order with that of EB-II and to understand the structural basis of memory effects observed in polyaniline; namely that when doped EB-I goes to ES-I and doped EB-II goes to ES-II.

\section{Experimental.}

2.1 NON-ENERGY DISPERSIVE DIFFRACTOMETRY WITH A PARALLEL BEAM. - In this technique a diffractometer has been adapted on a parallel $X$-ray beam. In that case the scattered radiation for very small Bragg angles, $\theta$, is not overshadowed by the direct beam which is considerably more intense than the diffracted beam. In order to separate fluorescence and incoherent radiations from the coherent component, $I_{\mathrm{c}}(K)$, the scattered beam is recorded by an energy dispersive detector which is connected to a preamplifier and a pulse processor. The pulse processor is a sophisticated signal processing unit which provides linear amplification, noise filtering, pulse pile-up rejection and life-time correction. The combination of these functions is an essential prerequisite for achieving accurate X-ray analysis. The pulses are accumulated in a multichannel analyzer interfaced with a computer. The solid state detector has an energy resolution of $150 \mathrm{eV}$ at $8 \mathrm{keV}$ and $230 \mathrm{eV}$ at $20 \mathrm{keV}$. Its high resolution in energy not only permit the elimination of fluorescence radiation from the sample but it also partially removes the incoherent Compton scattering. 
The experiment is mounted at a $\mathrm{Ag} \mathrm{X}$-ray source. In the present study only the $\lambda_{\mathrm{K} \alpha}=0.5609 \AA$ wavelength was used. The sample was scanned from $2 \theta=2^{\circ}$ to $90^{\circ}$ (i.e. from $K=0.4 \AA^{-1}$ to $15 \AA^{-1}$ ) in transmission geometry.

The effective elimination of the Compton scattering is better achieved by selecting the halfprofile of higher energy of the $\mathrm{Ag} \mathrm{K \alpha}$ characteristic spectrum. As the value of $K$ increases there is less and less overlap between the coherent and Compton profiles. For large $K$ values (above $7 \AA^{-1}$ in our case) the Compton peak can be totally separated and the upper half profile of $\mathrm{K} \alpha$ almost completely consists of the coherent component. In this respect the energy analysis performed has taken the place of an ideal monochromator in the diffracted beam. For smaller $K$ values this technique leads only to a partial removal of the Compton scattering. At low $K$ values (below $5 \AA^{-1}$ in our case) the Compton contribution to the $\mathrm{K} \alpha$ profile is negligibly small compared to the strong coherent intensity. There is thus a small range of $K$ values where the experimental data must be corrected in order to eliminate completely the Compton scattering. This has been done by a classical procedure of normalization. First the absolute value of the intensity of the interference function :

$$
J(K)=\frac{I_{c}(K)}{N f^{2}(K)}
$$

was obtained by scaling the atomic form factor $f(K)$ on the large $K$ data. Then small errors in the normalisation of $J(K)$, or more likely of the reduced interference function :

$$
F(K)=K[J(K)-1],
$$

were detected and corrected, with the help of the low $K$ data, in such a way that below the first correlation peak, the radial distribution function :

$$
W(r)=r(P(r)-1)=\left(2 \pi^{2} \rho_{0}\right)^{-1} \int_{0}^{\infty} F(K) \sin K r \mathrm{~d} K
$$

has a slope of -1 (i.e. below this peak there is a probability $P(r)=0$ to find an atom at a distance $r$ from a reference atom). Expressions (1) to (3) apply for a monoatomic isotropic amorphous material, of mean atomic volume $\rho_{0}^{-1}$ [1]. This is nearly the case of polyaniline emeraldine base $\left(\mathrm{C}_{24} \mathrm{H}_{18} \mathrm{~N}_{4}\right)$ because the carbon and nitrogen atoms have similar form factors and the $\mathrm{X}$-ray scattering by the hydrogen atoms is negligible.

2.2 SAMPLE. - The study has been performed on a $15 \times 10 \times 3 \mathrm{~mm}^{3}$ parallelepiped of EB-I pressed powder. Type I emeraldine base was prepared by the procedure outlined in reference [7].

\section{Experimental results and discussion.}

Figure 3 displays the reduced interference function $F(K)$ of EB-I. The $K_{\max }$ position of the first interference peak occurs at $\sim 1.4 \AA^{-1}$, which agrees with the maximum of the broad amorphous halo found in earlier investigations $[6,7]$ of EB-I materials. The reduced interference function of amorphous as prepared EB-II films exhibits the same $K_{\max }$ value, which also corresponds to approximately the position of the (110) reflection of the crystalline part of EB-II. This means that the average 1st interchain distance of amorphous EB-I and of as prepared EB-II are close to each other. A close comparison of figure 3 of this paper and of figure 3 of reference [4] shows however significant differences in the $K$ dependence of $F(K)$ in EB-I and in EB-II. 


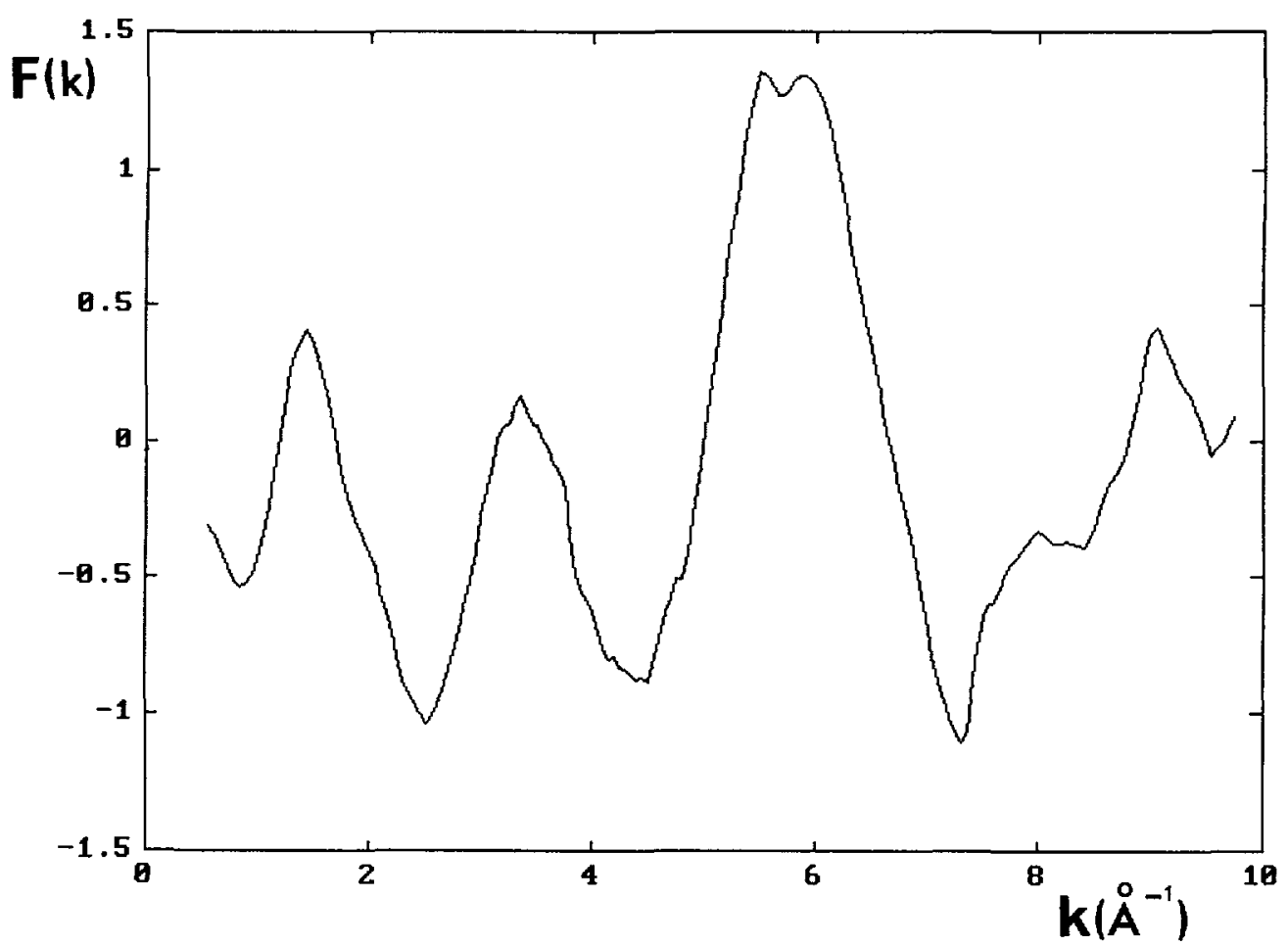

Fig. 3. - Reduced interference function $F(K)$ derived from the coherent part of the measured intensity $(\mathrm{Ag} \mathrm{K} \alpha)$ of EB-I.

The reduced radial distribution function of both materials also exhibits some differences. Figures $4 \mathrm{a}$ and $\mathrm{b}$ compare the $W(r)$ of EB-I and of as prepared EB-II respectively. Figure $4 \mathrm{a}$ is obtained through the expression (3) by the Fourier transform of $F(K)$ shown figure 3, and figure $4 \mathrm{~b}$ is taken from reference [4]. At the first interatomic distance and beyond it, positionposition correlations lead to oscillations of $P(r)$ and thus, from equation (3), to oscillations of $W(r)$. Figure 4 shows that these oscillations have about the same amplitude in EB-I and in as prepared EB-II, indicating that position-position correlations are of similar range. However the maxima of oscillations $\left(r_{\max }\right)$ are not exactly at the same positions in the two amorphous materials, which means that the average interatomic distance to the $n$-th neighbours slightly differs. As in the previous study of type II materials, it is useful to distinguish between the intrachain and interchain contributions of $W(r)$. For interatomic distances smaller than the Van der Waals diameter of a C atom, $d_{c}=3.4 \AA$ (Fig. 2a), only intrachain correlations contribute to the oscillations of $W(r)$. Calculations performed in reference [4] show that interchain correlations generally dominate for $r>d_{\mathrm{c}}$. For $r>d_{\mathrm{c}}$ the $W(r)$ of EB-I powder substantially differs from the $W(r)$ of dedoped unstretched ES-II film [4] by the amplitude of the oscillations and the $r_{\max }$ peak positions. EB-II obtained by the dedoping of amorphous ES-II exhibits a much stronger interchain disorder than EB-I obtained by the dedoping of partially crystalline ES-I. Such a difference in the range of position-position correlations observed after dedoping is probably related to the amorphous or substantially crystalline nature of the intermediate ES form [12]. The weak oscillations of $W(r)$ in dedoped unstretched ES-II film correspond to an interchain local order intermediate between those of ES-II and of EB-II [4].

Below $d_{c}, W(r)$ of EB-I shows 3 well defined peaks at $r_{\max }=1.4 \AA, 2.4 \AA$ and $3.2 \AA$ (Fig. 4a). The first distance agrees with the average 1st neighbor atomic distance of the 

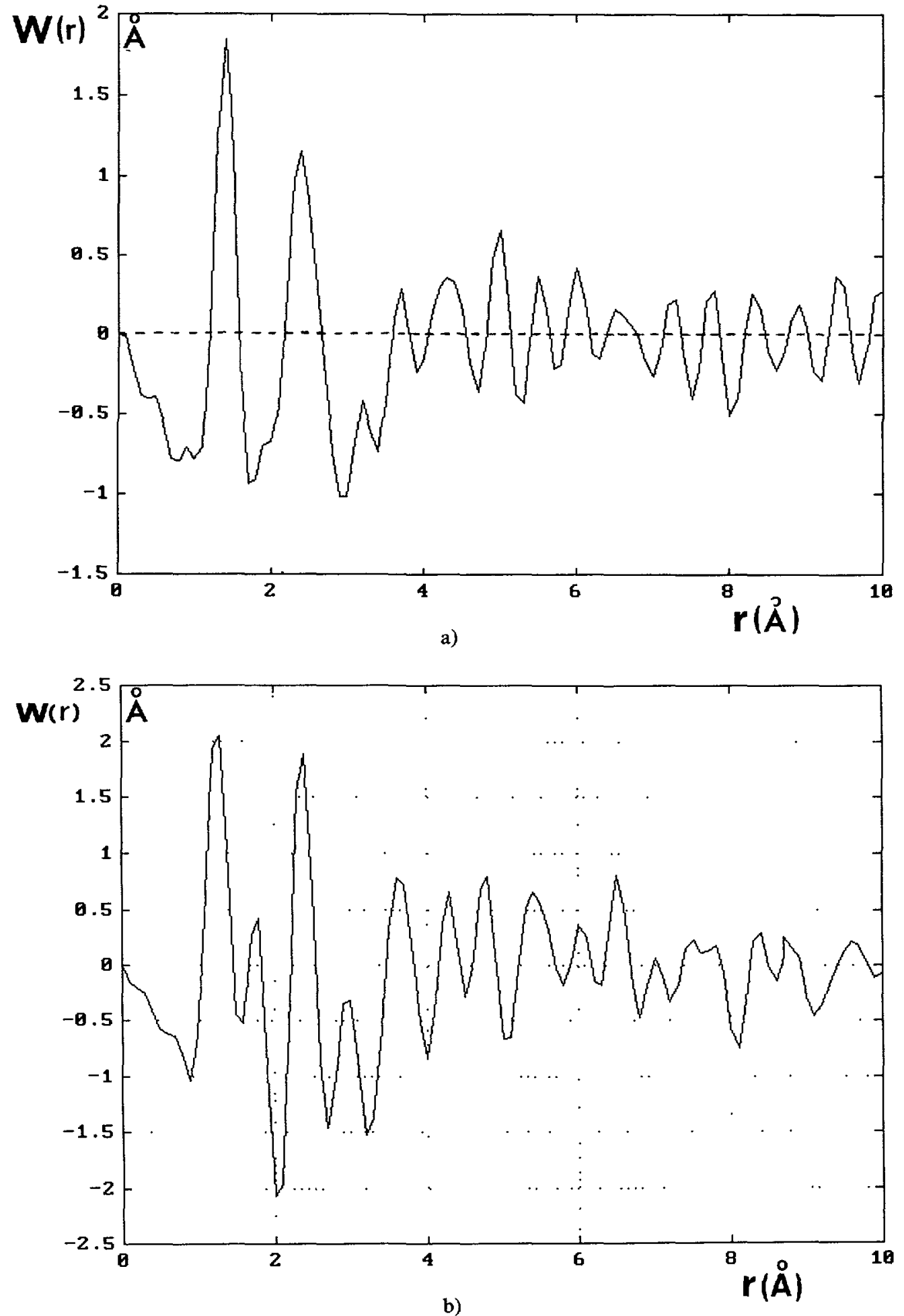

Fig. 4. - Reduced radial distribution function $W(r)$ of : (a) EB-I, derived from the Fourier transform of $F(K)$ shown in figure 3, (b) EB-II, according to reference [4]. 
polyaniline chain, shown figure $1, d=1.37_{5} \AA$, according to the structural refinements of salts of «tetramers» and «dimers» of aniline [8]. $2.4 \AA$ corresponds to the average 2 nd neighbor atomic distance of the chain, close to $d \sqrt{3}$. Beyond the 2nd neighbor there is a large spreading of intrachain distances essentially due to the distribution of ring tilt angles $\Phi$ and zig-zag angles $\delta$, the two main degrees of freedom of the polyaniline chain [4]. The corresponding $r_{\max }$ are located at $(1.25,1.8) \AA, 2.4 \AA$ and $2.9_{5} \AA$ in EB-II (Fig. 4b). The unphysical splitting of the 1st correlation peak of EB-II is believed to be caused by an artifact due to imperfect Compton scattering corrections in reference [4]. The 2nd correlation peak is found, as expected, at the same position in EB-I and EB-II. The 3rd correlation peak has a different position, which certainly indicate that the polymer chain adopts a slightly different conformation in the two amorphous base materials. $W(r)$ of EB-I and EB-II show some slight differences for $r>d_{c}$. This is especially the case for $r$ around $5 \AA$. This could be due to some change in the relative orientation between chains in close contact (1-2 and 1-3 chains of Fig. 2a). Such a feature is expected if the conformation of the polymer chains differs in EB-I and in EB-II. Conversely the conformation of the chains cannot be identical in the two base materials if the EB-I chain array keeps some memory of the local order adopted in ES-I where, as shown by figure 2 , the relative orientation between chains differs from that in EB-II. A previous study of type I polyaniline [7] has shown that there is a continuous structural evolution from ES-I to EB-I upon dedoping. No comparison between the $r_{\max }$ of $W(r)$ of EB-I and calculated interchain distances for polyaniline adopting a local array intermediate between those of ES-I and EB-II has been done because the ES-I « crystalline » structure is too crudely known. It is however interesting to point out that the oscillations of $W(r)$ of EB-I, for $r<5.5 \AA$, bear some resemblance with those shown by the carbon black, which has a graphitic like local order [13].

The slight difference of local order shown by $W(r)$ in the two classes of base materials provides a structural basis of the memory effect observed in polyaniline where :

- doping of EB-I yields the ES-I structure,

- doping EB-II yields the ES-II structure and redoping dedoped ES-II gives back again the ES-II structure,

without any interchange between the Class I and Class II branches of materials. The conversion from Class I to Class II polyaniline is only achieved through the dissolving of the polyaniline EB, or by the introduction and removal of solvent into the polymer.

The differences in local order within amorphous EB-I and amorphous EB-II suggest that there will be differences in the rates of gas diffusion [9] through films of the two polymers and that they may have different rates of ion exchange with electrolytes during electrochemical processes [10]. Other properties such as charge conduction and processes involving $\mathrm{C}_{6}$ ring rotations about their $\mathrm{N}-\mathrm{N}$ axes (for example, in formation of charged polarons [11]) may be affected by differences in the local order in EB-I and EB-II. Comparative studies of Class I and Class II materials are in progress in order to measure these differences.

\section{Acknowledgment.}

This work was supported in part by an «Action Incitative CNRS-NSF ». Laboratoire de Physique des Solides is associated to the Centre National de la Recherche Scientifique. J. Yue, M. E. Jozefowicz and Z. Oblakowski are thanked for the sample preparation. 


\section{References}

[1] Guinier A., Théorie et Technique de la Radiocristallographie ( $3^{\text {rd }}$ Ed. Dunod, Paris, 1964).

[2] KaKudo M. and KaSAI N., X-Ray Diffraction by Polymers, (Copublished by Kodunshaltd, Tokyo and Elsevier Publishing Company Amsterdam, 1972);

Windle A. H., Pure and Applied Chemistry 57/11 (1985) 1627.

[3] Laridjani M. and Sadoc J. F., J. Phys. France 42 (1981) 1293.

[4] Laridjani M., Pouget J. P., Scherr E. M., MacDiarmid A. G., Jozefowicz M. E. and EPSTEjn A. J., Macromolecules (May 1992).

[5] See, for example, Proc. Int. Conf. on the Science and Technology of Synthetic Metals, Tubingen, Germany, Sept. 1990, (Synth. Met. 41-43 (1991)) ; Santa Fe, USA, June 1988 (Synth. Met. 2729 (1989)).

[6] Jozefowicz M. E., Laversanne R., Javadi H. H. S., Epstein A. J., Pouget J. P., Tang X. and MacDiarmid A. G., Phys. Rev. B 39 (1989) 12958.

[7] Pouget J. P., Jozefowicz M. E., Epstein A. J., TAng X. and MacDiarmid A. G., Macromolecules 24 (1991) 779.

[8] Baughman R. H., Wolf J. P., Eckhard M. and Shacklette L. W., Synth. Met. 25 (1988) 121.

[9] Anderson M. R., Mattes B. R., Reiss H. and Kaner R. B., Science 252 (1991) 1412.

[10] Genies E. M., Boyle A., Lapkowski M. and Tsintaris C., Synth. Met. 36 (1990) 139;

Villeret B. and NeChtscheIn M., Phys. Rev. Lett. 63 (1989) 1285.

[11] Ginder J. M. and EPSTEIn A. J., Phys. Rev. B 41 (1990) 10674.

[12] In this respect, it is interesting to point out that the recently determined radial distribution function $W(r)$ of amorphous EB-II powder, obtained from the dedoping of partly « crystalline » ES-II powder, presents oscillations of magnitude comparable to those of $W(r)$ of EB-I, obtained from the dedoping of partly "crystalline » ES-I powder. In addition the oscillations of $W(r)$, for $r \leqslant 5 \AA$, show that dedoped ES-II powder exhibits a local order very close to that found [4] in never doped EB-II unstretched film (LARIDJANI M. et al., to be published).

[13] Warren B. E., J. Chem. Phys. 2 (1934) 551. 